\title{
Predictores de tuberculosis pulmonar y experiencia con su diagnóstico molecular rápido
}

\author{
Pulmonary tuberculosis predictors and rapid molecular diagnosis \\ Preditores de tuberculose pulmonar e experiência com o diagnóstico molecular rápido
}

\author{
Matilde Outeda', Elizabeth Marchissio², Pilar Gadea³, \\ Ivana Labadie ${ }^{4}$, Alice Bica5 ${ }^{5}$, Verónica Seija ${ }^{6}$
}

\section{Resumen}

A nivel mundial se estima que en 2018 hubo alrededor de 10 millones de nuevos casos de tuberculosis (TBC). La detección molecular es una herramienta diagnóstica crecientemente utilizada para el diagnóstico de TBC. Los predictores de riesgo para TBC pulmonar son variados y varían de acuerdo a la población estudiada. Los objetivos del presente trabajo fueron: evaluar la performance de la detección de M. tuberculosis por la técnica Xpert MTB/RIF para el diagnóstico de TBC pulmonar y determinar los factores predictores de presencia de esta enfermedad en pacientes asistidos en el Hospital Pasteur de Montevideo. Se realizó un estudio descriptivo, observacional y transversal. Se incluyeron 254 pacientes, 68 con TBC pulmonar. La sensibilidad de la prueba Xpert ${ }^{\circledR}$ MTB/RIF para detectar M. tuberculosis fue $100 \%$ (IC 95\%: 91,2-100) y la especificidad 95,1\% (IC 95\%: 83,9-98,7). En el análisis multivariado se evidenció que los predictores independientes para presencia de TBC pulmonar fueron: contacto cercano con otro caso de TBC $(p<0,001)$, consumo de pasta base de cocaína $(p=0,006)$ y presentarse con adelgazamiento $(p<0,001)$. En suma, la prueba Xpert ${ }^{\circledR}$ MTB/RIF se comportó como una excelente herramienta diagnóstica en nuestra población con elevada prevalencia de TBC pulmonar. Los predictores independientes para esta enfermedad indican que en la población analizada las estrategias de control de esta enfermedad requieren un abordaje multidisciplinario.

Palabras clave: Tuberculosis

Mycobacterium tuberculosis

Diagnóstico molecular

Factores de riesgo

Key words: Tuberculosis

Mycobacterium tuberculosis

Molecular diagnostics

Risk factors

\footnotetext{
1. Asistente Depto. de Laboratorio de Patología Clínica. Hospital de Clínicas. Facultad de Medicina. Universidad de la República.

2. Depto. de Emergencia. Hospital Pasteur. ASSE.

3. Prof. Adj. Depto. de Laboratorio de Patología Clínica. Hospital de Clínicas. Facultad de Medicina. Universidad de la República.

4. Licenciada en Laboratorio Clínico. Ex Pasante de Laboratorio Central. Hospital Pasteur. ASSE.

5. Depto. de Emergencia. Hospital Pasteur. ASSE.

6. Prof. Agdo. Depto. de Laboratorio de Patología Clínica. Hospital de Clínicas. Facultad de Medicina. Universidad de la República. Ex Encargada de Sección Bacteriología. Laboratorio Central. Hospital Pasteur. ASSE.

Correspondencia: Dra. Verónica Seija. Hospital de Clínicas. Avda Italia s/n. Montevideo, Uruguay. Correo electrónico: veronicaseija@hc.edu.uy, vseija1@gmail.com

Los autores no presentan conflictos de intereses.

El protocolo de investigación fue aprobado por el Comité de Ética del Hospital Pasteur.

Recibido: 6/10/2020

Aprobado: $21 / 12 / 2020$

Attribution-NonCommercial 4.0 International (CC BY-NC 4.0)
} 


\section{Introducción}

La tuberculosis (TBC) es una enfermedad infecto-contagiosa curable y prevenible, producida por Mycobacterium tuberculosis. A nivel mundial se estima que en 2018 hubo alrededor de 10 millones de nuevos casos, cifra estable en los últimos años ${ }^{(1)}$. En las Américas, en 2018, la estimación de incidencia fue de 29 casos cada 100.000 habitantes ${ }^{(1)}$. En Uruguay la incidencia registrada de esta enfermedad viene en aumento en los últimos años. Según datos de la Comisión Honoraria de la Lucha Antituberculosa y Enfermedades Prevalentes (CHLA-EP), la incidencia de TBC en 2006 fue de 17,2 casos cada 100.000 habitantes, mientras en 2018 trepó a $28,6 / 100.000^{(2)}$, con una estimación de $33 / 100.000^{(1)}$. Cerca de $90 \%$ de los casos presentaron localización pulmonar ${ }^{(2)}$. Los predictores de riesgo para TBC pulmonar son variados y varían de acuerdo a la población estudiada $^{(3)}$.

El cultivo continúa siendo el estándar de oro para la confirmación de la presencia de enfermedad tuberculosa y es recomendable para realizar los estudios de susceptibilidad antimicrobiana. Sin embargo, la amplificación de ácidos nucleicos es una herramienta diagnóstica crecientemente utilizada y recomendada ${ }^{(4)}$.

Xpert ${ }^{\circledR}$ MTB/RIF es una prueba de amplificación de ácidos nucleicos en tiempo real que se lleva a cabo en la plataforma GeneXpert ${ }^{\circledR}$ y permite detectar simultáneamente Mycobacterium tuberculosis complex (integrado por especies emparentadas genéticamente: $M$. tuberculosis, M. bovis, M. africanum, M. microti y M. canettii, entre otras) y resistencia a rifampicina asociada a mutaciones en el gen $r p o \mathrm{~B}^{(5)}$. A partir de diciembre de 2010, la Organización Mundial de la Salud recomienda el uso de esta prueba para el diagnóstico rápido de $\mathrm{TBC}^{(6)}$.

Los objetivos del presente estudio fueron: evaluar la performance de la detección de M. tuberculosis por la técnica Xpert ${ }^{\circledR}$ MTB/RIF para el diagnóstico de TBC pulmonar y determinar los factores predictores de presencia de esta enfermedad en pacientes asistidos en el Hospital Pasteur de Montevideo.

\section{Material y método}

Se realizó un estudio descriptivo, observacional y transversal.

Población: se incluyeron pacientes mayores de 18 años que consultaron entre enero de 2014 y junio de 2015 por sintomatología compatible con TBC pulmonar en el Hospital Pasteur (HP), perteneciente a la Administración de los Servicios de Salud del Estado (ASSE), y en quienes el médico tratante indicó la búsqueda rápida de M. tuberculosis en muestras respiratorias. Esta se realizó con la prueba Xpert ${ }^{\mathbb{B}}$ MTB/RIF en plataforma GeneXpert IV ${ }^{\circledR}$.
Metodología: para el estudio de performance de la prueba Xpert ${ }^{\mathbb{B}}$ MTB/RIF se incluyó una sola muestra por paciente que correspondió a la primera muestra del tracto respiratorio (expectoración, secreción respiratoria o lavado bronquioloalveolar) recibida en la Sección de Microbiología del Laboratorio Central del Hospital Pasteur (Lab-HP). La prueba Xpert ${ }^{\mathbb{B}}$ MTB/RIF fue realizada de acuerdo a las recomendaciones del fabricante. La solicitud y el envío de muestras para procesamiento en el Laboratorio de CHLA-EP (LabCHLA-EP) se recomendó en todos los informes escritos realizados por el Lab-HP, pero no se derivaron muestras desde el mismo. Se consideró la técnica de cultivo como el estándar de oro por lo que se excluyó, para esta parte del estudio, aquellas muestras en la que no contábamos con resultados de cultivos realizados por el LabCHLA-EP. Se consideraron sólo las muestras recibidas por LabCHLA-EP dentro de los cinco días antes o después de la muestra procesada en Lab-HP. Se excluyeron del estudio de performance pacientes con muestras de cultivo positivas dentro de los tres meses previos a la realización de la prueba Xpert ${ }^{\circledR}$ MTB/RIF.

En el LabCHLA-EP a todas las muestras se las cultivó, según protocolos internos, en medios sólido (Loweinstein-Jensen) o líquido (método automatizado BACTEC MGIT 960), con incubaciones de hasta ocho semanas y 42 días, respectivamente ${ }^{(7)}$. Además, se realizó baciloscopía por medio de tinción de fluorescencia, utilizando como fluorocromo la auramina-O, siendo éste su método de screening para la baciloscopía. Los informes de las baciloscopías se realizaron de acuerdo a la escala observada en la tabla $1^{(8)}$.

Para la determinación de los factores predictores de TBC pulmonar se realizó un estudio de casos/control donde los casos estuvieron constituidos por pacientes en quienes se detectó $M$. tuberculosis en la prueba $\mathrm{Xpert}^{\mathbb{R}}$ MTB/RIF o cultivo. Los controles fueron pacientes con sospecha de TBC pulmonar, pero en quienes no se detectó M. tuberculosis. Los datos clínicos se recogieron en un formulario previamente elaborado que incluyó el reporte de características clínicas y epidemiológicas del paciente, las cuales están consignadas en la tabla 4. Este formulario se encontraba disponible en todas las áreas del hospital y era completado por el médico que solicitaba el estudio.

Para el análisis de los factores de riesgo se incluyeron aquellos pacientes en quienes el formulario de solicitud estaba correctamente completado por el médico tratante y acompañado de una muestra clínica de origen respiratorio.

\section{Definiciones}

Se definió la presencia de contacto cercano en aquellas personas los contactos convivientes o no convivientes 
Tabla 1. Escala de lectura de baciloscopía con tinción fluorescente (auramina-O) CHLA-EP, 2018.

\begin{tabular}{ll}
\hline Resultado del examen microscópico a $400 x$ & Informe \\
\hline $\begin{array}{l}\text { No se encuentran BAAR en los } \\
100 \text { campos observados }\end{array}$ & No BAAR \\
1-9 BAAR en 100 campos & Positivo \\
10-99 BAAR en 100 campos & Positivo $(+)$ \\
1-10 BAAR por campo & Positivo $(++)$ \\
$>10$ BAAR por campo & Positivo $(+++)$ \\
\hline
\end{tabular}

BAAR: bacilos ácido alcohol resistentes.

pero que compartieron un espacio cerrado (laboral o de estudio) por extensos períodos con una persona con TBC conocida, cualesquiera fueran las características de la enfermedad: localización (pulmonar o extrapulmonar) y categoría diagnóstica (confirmada o no confirmada bacteriológicamente) en los tres meses previos al inicio del tratamiento ${ }^{(8)}$.

Se interrogaron y registraron los siguientes factores de riesgo: sexo, edad, síntomas respiratorios, diabetes, insuficiencia renal crónica, neoplasia, infección por virus de inmunodeficiencia adquirida, tabaquismo, consumo de marihuana, consumo de pasta base de cocaína (PBC), alcoholismo. La identificación de éstos quedó a cargo del médico tratante. No se realizaron estudios adicionales que podrían haber detectado nuevas situaciones de complicación al momento de la consulta.

Ética: el protocolo de investigación fue aprobado por el Comité de Ética del Hospital Pasteur. El manejo de los datos obtenidos se realizó manteniendo el anonimato de los individuos.

Análisis estadístico: la comparación de variables continuas se realizó mediante el test de Student. La relación entre variables categóricas se estudió mediante la prueba de $\mathrm{X}^{2} \mathrm{y}$ el test exacto de Fisher, según correspondiera. Para la performance diagnóstica se calculó sensibilidad, especificidad y valores predictivos. Se expresaron en porcentaje e intervalo de confianza de $95 \%$ (IC 95\%). Se utilizaron coeficientes de correlación para comparar la semicuantificación de baciloscopía y prueba Xpert ${ }^{\circledR}$ MTB/RIF. Se realizó análisis multivariado para determinar los factores predictores independientes de TBC pulmonar. El valor de significación fue 0,05.

\section{Resultados}

Se incluyeron 254 pacientes con sospecha de TBC pulmonar, $163(64,2 \%)$ de sexo masculino. La media de edad fue $49,36 \pm 18,54$ y $47,15 \pm 17,76$ para el sexo
Tabla 2. Resultados Xpert ${ }^{\circledast}$ MTB/RIF y cultivo de $M$. tuberculosis.

\begin{tabular}{lccc|}
\hline & $\begin{array}{c}\text { Cultivo desarrolla } \\
\text { M. tuberculosis }\end{array}$ & $\begin{array}{c}\text { Cultivo no } \\
\text { desarrolla } \\
\text { M. tuberculosis, }\end{array}$ & Total \\
\hline $\begin{array}{l}\text { Xpert }{ }^{\circledR} \text { MTB/RIF } \\
\text { positivo }^{\text {a }}\end{array}$ & 40 & 2 & 42 \\
$\begin{array}{l}\text { Xpert }{ }^{\circledR} \text { MTB/RIF } \\
\text { negativo }\end{array}$ & 0 & 39 & 39 \\
Total & 40 & 41 & 81 \\
\hline $\begin{array}{l}\text { a El resultado positivo corresponde a detección de M. tuberculosis. } \\
\text { No se detectó resistencia a rifampicina. }\end{array}$ \\
\hline
\end{tabular}

masculino y femenino, respectivamente. La procedencia de las muestras fue: $28(11 \%)$ de la unidad de cuidado intensivo; $69(27,2 \%)$ de cuidados moderados y 157 $(61,8 \%)$ del Departamento de Emergencia.

Para el estudio de performance de la prueba Xpert ${ }^{\circledR}$ MTB/RIF se obtuvo el resultado del cultivo para $M$. $t u$ berculosis del LabCHLA-EP en 84 pacientes (33,1\%). Para evaluar la prueba diagnóstica se excluyeron tres muestras con prueba Xpert ${ }^{\circledR}$ MTB/RIF positiva: una porque el cultivo se informó como contaminado y dos porque los pacientes tenían confirmación microbiológica de TBC pulmonar dentro de los tres meses previos. Los resultados de los cultivos de los 81 pacientes restantes en relación con la prueba Xpert ${ }^{\mathbb{B}}$ MTB/RIF se muestran en la tabla 2. En ninguna muestra se detectó resistencia a rifampicina en la prueba $\mathrm{Xpert}^{\mathbb{B}}$ MTB/RIF, ni en los cultivos del LabCHLA-EP. La sensibilidad de la prueba Xpert ${ }^{\mathbb{B}}$ MTB/RIF para detectar M. tuberculosis fue 100\% (IC 95\%: 91,2-100) y la especificidad 95,1\% (IC 95\%: 83,9-98,7). Las dos muestras con prueba Xpert ${ }^{\mathbb{B}}$ MTB/RIF positiva y cultivo negativo no manifestaron tener antecedentes de TBC, ni tampoco se evidenció confirmación microbiológica de TBC pulmonar en resultados de LabCHLA-EP. En nuestra población, el valor predictivo positivo de la prueba Xpert ${ }^{\circledR}$ MTB/RIF fue $95,2 \%$ (IC 95\%: $84,1-98,7$ ) y el valor predictivo negativo $100 \%$ (IC 95\%: 91,0-100).

En 40/44 de las muestras que mostraron prueba Xpert ${ }^{\circledR}$ MTB/RIF positiva, se registró el resultado de la carga semicuantitativa de ADN que informa la plataforma GeneXpert. En la tabla 3 se puede observar la relación entre este resultado y el de escala de la baciloscopía que informó el LabCHLA-EP.

En el estudio de casos/control se incluyeron 254 pacientes, 68 casos y 186 controles. 
Tabla 3. Resultado semicuantitativo de Xpert ${ }^{\circledR}$ MTB/RIF versus semicuantificación en baciloscopía

\begin{tabular}{|c|c|c|c|c|c|c|}
\hline \multirow{2}{*}{$\begin{array}{c}\text { Carga ADN } \\
\text { informada en } \\
\text { Xpert }{ }^{\circledR} \text { MTB/RIF }\end{array}$} & \multicolumn{6}{|c|}{ Resultado baciloscopia en Laboratorio CHLA-EP } \\
\hline & Positiva (< a 5 BAAR) & Positiva (+) & Positiva (++) & Positiva (+++) & Negativa & Total \\
\hline Alta & 0 & 0 & 1 & 8 & 0 & 9 \\
\hline Media & 0 & 3 & 5 & 9 & 2 & 19 \\
\hline Baja & 1 & 0 & 5 & 1 & 4 & 11 \\
\hline Muy baja & 0 & 0 & 0 & 0 & 1 & 1 \\
\hline Total & 1 & 3 & 11 & 18 & 7 & 40 \\
\hline
\end{tabular}

$R$ de Pearson: 0,58, $\mathrm{p}<0,001$. Correlación de Spearman: 0,60, $\mathrm{p}<0,001$

En la tabla 4 se muestran los resultados obtenidos de cada variable incluida en el estudio: edad, sexo, contacto cercano, antecedentes personales patológicos y manifestaciones clínicas. En el análisis multivariado se evidenció que los predictores independientes para presencia de TBC pulmonar fueron: contacto cercano con TBC ( $<<0,001$, OR 4,469, IC 95\%: 2,254-8,861), consumo de PBC ( $\mathrm{p}=0,006$, OR 2,820 IC 95\%: 1,349-5,891) y que se presentaran con adelgazamiento $(\mathrm{p}<0,001$, OR 3,395 IC 95\%: 1,766-6,528). En el resto de las variables la asociación no fue estadísticamente significativa en el análisis multivariado.

\section{Discusión}

En relación con la técnica de Xpert ${ }^{\mathbb{B}}$ MTB/RIF para el diagnóstico de TBC pulmonar, se demostró una excelente sensibilidad. No se detectaron casos con cultivos positivos y Xpert ${ }^{\circledR}$ MTB/RIF negativo. Este hecho llamó la atención, ya que diferentes series demuestran una disminución de sensibilidad de esta prueba en muestras con baciloscopía negativa ${ }^{(9-11)}$. En nuestra serie, hubo muestras con cultivo positivo y baciloscopía negativa, pero estas fueron detectadas por la prueba $\mathrm{Xpert}^{\mathrm{B}}$ MTB/RIF. El bajo número de este tipo de muestras podría explicar, en parte, nuestros resultados.

La especificidad fue muy buena, solo dos en 42 muestras positivas con Xpert ${ }^{\mathbb{B}}$ MTB/RIF tuvieron cultivo negativo. La cifra de especificidad encontrada en nuestra serie es similar a la de otros trabajos ${ }^{(9,12)}$. Nuestro número de falsos positivos fue muy bajo y en estos dos casos no encontramos evidencia de TBC en sus antecedentes.

En el estudio de performance, decidimos excluir dos pacientes con antecedentes de TBC, ya que se ha demostrado la persistencia de Xpert ${ }^{\circledR}$ MTB/RIF positivo en hasta $30 \%$ de los pacientes que completaron seis meses de tratamiento y presentaron cura microbiológica (baciloscopía y cultivo negativo) ${ }^{(13)}$. Esto apoya la recomendación de no utilizar la prueba Xpert ${ }^{\mathbb{Q}}$ MTB/RIF para el control de la respuesta al tratamiento en pacientes con $\mathrm{TBC}^{(8)}$. Theron y colaboradores evaluaron el significado de 16 falsos positivos encontrados en 238 pacientes con antecedentes de TBC y una media de 11 meses luego de finalizado el tratamiento ${ }^{(14)}$. Para ello, realizaron un seguimiento clínico y paraclínico en el cual encontraron que estos pacientes se mantuvieron sanos y la mitad presentó una nueva prueba Xpert ${ }^{\circledR}$ MTB/RIF negativa. Concluyeron que en los pacientes con antecedentes de TBC existe la posibilidad de falsos positivos con Xpert ${ }^{\mathbb{B}}$ MTB/RIF y que estos pacientes deben ser evaluados cuidadosamente.

Con respecto a la cuantificación de ADN bacilar que informa Xpert ${ }^{\mathbb{B}}$ MTB/RIF, su valor es relativo y no se recomienda informar esta cuantificación. Esta depende de muchos factores, como tipo, volumen y calidad de la muestra, centrifugación previa, etc. En nuestro caso hubo una importante correlación entre esta cuantificación y la escala de cuantificación de la baciloscopía realizada por el LabCHLA-EP. Otro hecho a destacar es que la mayor parte de las baciloscopías se presentaron con dos y tres cruces de positividad indicando que en la población estudiada los diagnósticos fueron tardíos.

En 2014, año de nuestro trabajo, la tasa de incidencia de TBC fue de 25,2 casos cada 100.000 habitantes $^{(15)}$, concentrados en Montevideo, con una incidencia de 38,3/100.000. Los casos de Montevideo se concentran en los municipios A, D, F y G ${ }^{(2)}$, donde se alcanzan cifras de incidencia de 50 casos/100.000. El Hospital Pasteur atiende alrededor de 200.000 usuarios, tanto de Montevideo como del interior. Por su localización geográfica, podemos presumir que la mayoría de sus usuarios de Montevideo provienen de los municipios D, E y F. Los 
Tabla 4. Factores predictores para tuberculosis pulmonar: análisis uni y multivariado.

\begin{tabular}{|c|c|c|c|c|c|}
\hline Variable & $\begin{array}{c}\text { Casos } \\
N=68 \\
(26,8 \%)\end{array}$ & $\begin{array}{c}\text { Controles } \\
N=186 \\
(73,2 \%)\end{array}$ & Valor $p$ & $\begin{array}{l}\text { OR ajustado } \\
\text { (IC 95\%) }\end{array}$ & Valor $p$ \\
\hline Sexo masculino & $43(63,2 \%)$ & $120(64,5 \%)$ & 0,850 & & \\
\hline Menor de 40 años & $36(52,9 \%)$ & $59(23,7 \%)$ & 0,003 & & \\
\hline \multicolumn{6}{|c|}{ Síntomas al momento de la consulta } \\
\hline Tos & $56(82,4 \%)$ & $160(86,0 \%)$ & 0,468 & & \\
\hline Expectoración & $52(76,5 \%)$ & $153(82,3 \%)$ & 0,301 & & \\
\hline Hemoptisis & $12(17,3 \%)$ & $23(12,4 \%)$ & 0,280 & & \\
\hline Sudoración nocturna & $40(58,8 \%)$ & $72(38,7 \%)$ & 0,004 & & \\
\hline Fiebre & $42(61,8 \%)$ & $89(47,8 \%)$ & 0,490 & & \\
\hline Adelgazamiento & $45(66,2 \%)$ & $67(36,0 \%)$ & $<0,001$ & $\begin{array}{c}3,395 \\
(1,766-6,528)\end{array}$ & $<0,001$ \\
\hline Disnea & $28(41,2 \%)$ & $78(41,9 \%)$ & 0,914 & & \\
\hline Anorexia & $27(39,7 \%)$ & $38(20,4 \%)$ & 0,002 & & \\
\hline \multicolumn{6}{|l|}{ Antecedentes } \\
\hline Contacto cercano & $31(45,6 \%)$ & $36(19,4 \%$ & $<0,001$ & $\begin{array}{c}4,469 \\
(2,254-8,861)\end{array}$ & $<0,001$ \\
\hline Tuberculosis previa & $23(33,8 \%)$ & $34(18,3 \%)$ & 0,009 & & \\
\hline Internación año previo & $17(25,0 \%)$ & $58(31,2 \%)$ & 0,339 & & \\
\hline Internación mes previo & $5(7,4 \%)$ & $18(9,7 \%)$ & 0,568 & & \\
\hline Consumo PBC & $25(36,8 \%)$ & $23(12,4 \%)$ & $<0,001$ & $\begin{array}{c}2,820 \\
(1,349-5,891)\end{array}$ & 0,006 \\
\hline Consumo marihuana & $12(17,6 \%)$ & $8(4,3 \%)$ & $<0,001$ & & \\
\hline Tabaquismo & $45(66,2 \%)$ & $109(58,6 \%)$ & 0,274 & & \\
\hline Alcoholismo & $14(20,6 \%)$ & $37(19,9 \%)$ & 0,902 & & \\
\hline Portador de VIH & $12(17,6 \%)$ & $38(20,4 \%)$ & 0,621 & & \\
\hline Diabetes mellitus & $5(7,4 \%)$ & $15(8,1 \%)$ & 0,852 & & \\
\hline Insuficiencia renal crónica & $3(4,4 \%)$ & $9(4,8 \%)$ & 0,887 & & \\
\hline Neoplasia & $1(1,5 \%)$ & $4(2,2 \%)$ & 0,730 & & \\
\hline
\end{tabular}

municipios D y F ocupan el segundo y tercer puesto, respectivamente, en el número de casos detectados. Esto podría explicar la alta frecuencia de muestras positivas encontradas $(68 / 254,26,8 \%)$.

Llamó la atención que solo en 84 de 254 muestras se obtuvo resultado de cultivo en el LabCHLA-EP. Aunque esto podría ser debido a errores preanalíticos de identificación de pacientes o muestras, es claro que muchas veces no hubo envío a CHLA-EP, confiando en el resultado emitido por Xpert ${ }^{\mathbb{R}}$ MTB/RIF. Esto es riesgoso, ya que aunque nosotros encontramos muy buenos valores predictivos positivo y negativo, aún puede haber muestras con baciloscopía y Xpert ${ }^{\circledR}$ MTB/RIF negativo que tengan un cultivo positivo. En este sentido, Rasheed y colaboradores ${ }^{(16)}$ reportaron $27 / 78$ muestras con estas características. Por otro lado, el no remitir las muestras impide que se realicen los estudios de susceptibilidad antimicrobiana. En nuestra serie no hubo Xpert ${ }^{\mathbb{B}}$ MTB/RIF con señal positiva para resistencia a rifampicina, ni cultivos con $M$. tuberculosis resistente a rifampicina. Esto es consistente con las bajas cifras de resistencia a rifampicina que se reportan en nuestro país ${ }^{(2)}$.

Detectamos 68 casos con prueba Xpert ${ }^{\mathbb{B}}$ MTB/RIF positiva, 23 declararon haber tenido TBC previa. Para el 
estudio de factores de riesgo, los consideramos casos, pero es claro que podrían representar recaídas. De estos 68 casos, solo $40(58,8 \%)$ fueron confirmados por cultivo, una tasa muy por debajo de la tasa de confirmados $(73,8 \%)$ que reporta la CHLA-EP en $2014^{(15)}$.

Se evaluaron algunos de los predictores para presentar TBC pulmonar y de los evaluados, no todos fueron exhaustivamente investigados. Los predictores independientes para presentar TBC pulmonar fueron, dentro de la presentación clínica, adelgazamiento y luego ser contacto cercano de una persona con TBC y consumir PBC. El adelgazamiento es uno de los síntomas clásicos dentro de los reportados asociados a la TBC pulmonar. En nuestro estudio fue el único síntoma que predijo en forma independiente la presencia de TBC pulmonar. Un metaanálisis que investigó 11 estudios, encontró el adelgazamiento como predictor de riesgo independiente para TBC pulmonar ${ }^{(3)}$. Una historia de tos y expectoración persistente podrían ser buenos predictores en pacientes que consultan en servicios de atención primaria. Sin embargo, no son buenos predictores en pacientes que recurren al hospital, tal como ha sido previamente reportado por Greenaway y colaboradores ${ }^{(17)}$.

El ser contacto cercano de otro caso es un factor de riesgo conocido y estudiado desde hace mucho tiempo. El riesgo va en relación con la carga bacteriana y el tiempo de contacto. Es claro que los pacientes con baciloscopía positiva tienen mayor capacidad de transmisión ${ }^{(3)}$. Morrison y colaboradores hicieron una revisión y encontraron que $4,5 \%$ de los contactos desarrollaron TBC confirmada clínica y microbiológicamente ${ }^{(18)}$. Asimismo, 51,4\% de los contactos fueron detectados con infección tuberculosa latente. En nuestra serie, 45,6\% de los casos declaró ser contacto cercano de un paciente con TBC y esto multiplicó por cuatro su riesgo para desarrollar TBC pulmonar. Estos datos indican que es fundamental intensificar las acciones para buscar los contactos y realizar la quimioprofilaxis, cuando corresponda, para disminuir los casos de TBC. En cuanto a los predictores relacionados con el individuo destacamos que, al contrario de lo que se reporta ${ }^{(3)}$, en la población estudiada, tabaquismo, alcoholismo, diabetes e infección por VIH no estuvieron relacionados con el desarrollo de TBC. Esto podría ser debido a que nuestro estudio fue un corte transversal y se interrogó al paciente en una sola oportunidad. A pesar de ello, el uso de PBC multiplicó casi por tres el riesgo de TBC pulmonar. La PBC es un producto intermediario en la producción del clorhidrato de cocaína. Según estudios locales ${ }^{(19)}$, la mayoría de las muestras de PBC contienen entre $30 \%$ y 50\% de cocaína base, siendo fenacetina y cafeína los otros componentes más frecuentes. Al igual que la $\mathrm{PBC}$, el crack es otro ejemplo de cocaína fumable, pero se trata de un produc- to diferente a la PBC, derivado de la cocaína en polvo. De manera interesante, los consumidores de crack poseen un perfil clínico similar a los de $\mathrm{PBC}^{(20)}$. El uso de drogas ilícitas ha sido ampliamente relacionado con el desarrollo de $\mathrm{TBC}^{(21)}$. Varios estudios encontraron el consumo de crack como un factor de riesgo para la infección tuberculosa latente (ITL) y también para enfermedad tuberculosa ${ }^{(22-24)}$, llegando a multiplicar por seis el riesgo de adquirir TBC bacilifera ${ }^{(25)}$. Sin embargo, no se encontraron trabajos que establezcan el consumo de $\mathrm{PBC}$ como factor de riesgo para TBC. Este riesgo aumentado que presenta el consumo de $\mathrm{PBC}$ en nuestro estudio podría deberse a los efectos que tiene la cocaína sobre el pulmón, tales como la disminución en la función de los macrófagos alveolares y producción de cioquinas, entre otros ${ }^{(26,27)}$. Es claro que este riesgo aumentado es también debido a los determinantes sociales asociados al consumo de PBC en nuestro país ${ }^{(28)}$. Pensamos, entonces, que esta asociación estadísticamente significativa entre TBC pulmonar y consumo de PBC debe ser considerada dentro de este contexto para poder analizar el problema en todas sus dimensiones. Otro elemento no menor, a la hora de diseñar estrategias de control, es que los estudios marcan el uso de drogas ilícitas como un predictor para abandono de tratamiento y resistencia antibiótica en $M$. tuberculosis ${ }^{(29,30)}$.

En suma, la prueba Xpert ${ }^{\mathbb{B}}$ MTB/RIF se comportó como una excelente herramienta diagnóstica en nuestra población con elevada prevalencia de TBC pulmonar. Los predictores independientes para esta enfermedad fueron: adelgazamiento, ser contacto cercano de otra persona con TBC y utilizar PBC. Por ello, en la población analizada, las estrategias de control de esta enfermedad deben ser estudiadas a la luz de estos resultados. Sin duda, se requiere un abordaje multidisciplinario del problema.

\section{Agradecimientos}

A todos los médicos e internos del Hospital Pasteur por el tiempo que se tomaron en completar el protocolo que sirvió de base para este estudio.

\section{Summary}

According to global estimations, there were approximately 10 million new cases of tuberculosis in 2018. Molecular diagnosis constitutes a rapidly growing diagnostic tool for tuberculosis. Risk predictors for pulmonary tuberculosis are varied and they depend on the population studied.

The study aimed to assess the performance of $M$. tuberculosis detection by use of Xpert ${ }^{\circledR}$ MTB/RIF diagnostic test to diagnose pulmonary tuberculosis and to identify predictive factors for this disease in patients assisted at Pasteur Hospital in Montevideo. 
A descriptive, observational and transversal study was conducted, which included 254 patients, 68 of which had pulmonary tuberculosis. Sensitivity of the Xpert MTB/RIF assay to detect M. tuberculosis was 100\% (CI 95\%: 91.2-100) and specificity 95.1\% (CI 95\%: 83.9-98.7). Multivariate analysis evidenced the following to be the independent predictors that detect pulmonary tuberculosis: close contact with other cases of tuberculosis $(\mathrm{p}<0.001)$, coca-paste consumption $(p=0.006)$ and evidence of loss of weight $(p<0,001)$.

To sum up, the Xpert ${ }^{\mathbb{B}}$ MTB/RIF assay proved to be an excellent diagnostic tool in our population with a high prevalence of pulmonary tuberculosis. Independent predictors for this disease show that, in the population studied, control strategies require a multidisciplinary approach.

\section{Resumo}

Globalmente, estima-se que em 2018 ocorreram cerca de 10 milhões de novos casos de tuberculose (TB). A detecção molecular é uma ferramenta diagnóstica cada vez mais usada para seu diagnóstico. Os preditores de risco para TB pulmonar são diversos e variam de acordo com a população estudada.

Os objetivos deste estudo foram: avaliar o desempenho da detecção do M. tuberculosis pela técnica Xpert MTB/RIF para o diagnóstico da TB pulmonar e determinar os fatores preditivos da presença desta doença em pacientes atendidos no Hospital Pasteur de Montevidéu.

Foi realizado um estudo descritivo, observacional e transversal. 254 pacientes foram incluídos, 68 com TB pulmonar. A sensibilidade do teste Xpert ${ }^{\circledR}$ MTB/RIF para detectar $M$. tuberculosis foi de 100\% (IC 95\%: 91,2-100) e a especificidade de 95,1\% (IC 95\%: 83,998,7). A análise multivariada mostrou que os preditores independentes para a presença de tuberculose pulmonar foram: contato próximo com outro caso de tuberculose ( $\mathrm{p}<0,001)$, consumo de pasta base de cocaína $(\mathrm{p}=$ $0,006)$ e apresentar perda de peso $(\mathrm{p}<0,001)$.

Em suma, o teste Xpert ${ }^{\circledR}$ MTB/RIF se comportou como uma excelente ferramenta diagnóstica em nossa população com alta prevalência de TB pulmonar. Os preditores independentes para essa doença indicam que, na população analisada, as estratégias de controle da doença requerem uma abordagem multidisciplinar.

\section{Bibliografía}

1. World Health Organization. Global tuberculosis report 2019. WHO, 2019. Disponible en: https://apps.who.int/iris/ bitstream/handle/10665/329368/9789241565714eng.pdf?ua=1. [Consulta: 22 agosto 2020].

2. Comisión Honoraria para la Lucha Antituberculosa y Enfermedades Prevalentes. Informe tuberculosis 2018. CHLA-EP, Montevideo. Disponible en: http://chlaep.
org.uy/wp-content/uploads/2019/05/Situaci\%C3\%B3n-Tuberculosis-Uruguay-2018.pdf. [Consulta: 2 agosto 2020].

3. Narasimhan P, Wood J, Macintyre CR, Mathai D. Risk factors for tuberculosis. Pul Med 2013; 828939. doi: $10.1155 / 2013 / 828939$.

4. Diagnosis of tuberculosis disease. En: Centers for Disease Control and Prevention. Core curriculum on tuberculosis: what the clinician should know. CDC, 2013: 75-108.

5. World Health Organization. Automated real-time nucleic acid amplification technology for rapid and simultaneous detection of tuberculosis and rifampicin resistance: Xpert MTB/RIF assay for the diagnosis of pulmonary and extra-pulmonary TB in adults and children. Policy update. Geneva: WHO, 2013. Disponible en: https://apps. who.int/iris/bitstream/handle/10665/112472/ 9789241506335_eng.pdf?sequence $=1$ [Consulta: 2 agosto 2020].

6. World Health Organization. Rapid implementation of the Xpert MTB/RIF diagnostic test. Technical and operational 'How-to' practical considerations. Geneva: WHO, 2011. Disponible en: https://www.who.int/tb/publications/tb-amplificationtechnology-implementation/en/ [Consulta: 28 julio 2020].

7. Organización Panamericana de la Salud. Manual para el diagnóstico bacteriológico de la tuberculosis. Normas y guía técnica. Parte II: Cultivos. OPS, 2008. Disponible en: https://iris.paho.org/bitstream/handle/10665.2/18616/tblabscultivo_2008.pdf?sequence $=1 \&$ isAllowed $=y \quad$ [Consulta: 2 agosto 2020].

8. Guía nacional para el manejo de la tuberculosis. Ministerio de Salud, CHLA-EP, Facultad de Medicina. Montevideo, 2016. Disponible en: http://www.chlaep.org.uy/descargas/programas-control-tuberculosis/normas/guia-nacional-para-el-manejo.pdf [Consulta: 2 agosto 2020].

9. Vallejo P, Rodríguez JC, Searle A, Farga V. Xpert MTB/RIF en el diagnóstico de tuberculosis. Rev Chil Enf Respir 2015; 31: 127-131. doi: 10.4067/S0717-73482015000200010.

10. Helb D, Jones M, Story E, Boehme C, Wallace E, Ho K, et al. Rapid detection of mycobacterium tuberculosis and rifampin resistance by use of on-demand, near-patient technology. J Clin Microbiol 2010; 48:229-37. doi: 10.1128/JCM. 01463-09.

11. Blakemore R, Story E, Helb D, Kop JA, Banada P, Owens MR, et al. Evaluation of the analytical performance of the Xpert MTB/RIF Assay. J Clin Microbiol 2010; 48:2495-501. doi: 10.1128/JCM.00128-10.

12. Zeka AN, Tasbakan S, Cavusoglu C. Evaluation of the GeneXpert MTB/RIF assay for rapid diagnosis of tuberculosis and detection of rifampin resistance in pulmonary and extrapulmonary specimens. J Clin Microbiol 2011; 49:4138-41. doi: 10.1128/JCM.05434-11.

13. Friedrich SO, Rachow A, Saathoff E, Singh, K, Mangu CD, Dawson R, et al. Assessment of the sensitivity and specificity of Xpert MTB/RIF assay as an early sputum biomar- 
ker of response to tuberculosis treatment. Lancet Respirator Med 2013;1:462-70. doi: 10.1016/s2213-2600(13)70119-x.

14. Theron G, Venter R, Smith L, Esmail A, Randall P, Sood $\mathbf{V}$, et al. False-positive Xpert MTB/RIF results in retested patients with previous tuberculosis: frequency, profile, and prospective clinical outcomes. J Clin Microbiol 2018; 56:e1696-17. doi: 10.1128/JCM.01696-17.

15. Comisión Honoraria para la Lucha Antituberculosa y Enfermedades Prevalentes. Programa Nacional de Control de la Tuberculosis. Informe Año 2014. Cifras Definitivas. Montevideo: CHLA-EP, 2014. Disponible en: http://www.chlaep.org.uy/descargas/programas-control-tuberculosis/estadisticas/2014-cifras-definitivas.pdf C [Consulta: 2 agosto 2020].

16. Rasheed W, Rao NA, Adel H, Baig MS, Adil SO. Diagnostic accuracy of xpert MTB/RIF in sputum smear-negative pulmonary tuberculosis. Cureus 2019; 11(8):e5391. doi: 10.7759/cureus.539.

17. Greenway C, Menzies D, Fanning A, Grewal R, Yuan L, Fitzgerald JM, et al. Delay in diagnosis among hospitalized patients with active tuberculosis - predictors and outcomes. Am J Respir Crit Care Med 2002; 165:927-33. doi: 10.1164/ajrccm.165.7.2107040.

18. Morrison J, Pai M, Hopewell PC. Tuberculosis and latent tuberculosis infection in close contacts of people with pulmonary tuberculosis in low-income and middle-income countries: a systematic review and meta-analysis. Lancet Infect Dis 2008; 8:359-68. doi: 10.1016/S1473-3099(08)70071-9.

19. Abin-Carriquiry JA, Martínez-Busi M, Galvalisi M, Minteguiaga M, Prieto JP, Scorza MC. Identification and quantification of cocaine and active adulterants in coca-paste seized samples: useful scientific support to health care. Neurotox Res 2018; 34(2):295-304. doi: 10.1007/s12640018-9887-1.

20. Moraes M, Scorza C, Abin-Carriquiry JA, Pascale A, González G, Umpiérrez E. Consumo de pasta base de cocaína en Uruguay en el embarazo, su incidencia, características y repercusiones. Arch Ped Urug 2010; 81:100-4. Disponible en: http://www.scielo.edu.uy/scielo.php?script=sci_arttext\&pid=S1688-12492010000200006 [Consulta: 2 agosto 2020].

21. Deiss RG, Rodwell TC, Garfein RS. Tuberculosis and illicit drug use: review and update. Clin Infect Dis 2009; 48:72-82. doi: $10.1086 / 594126$.
22. Leonhardt KK, Gentile F, Gilbert BP, Aiken M. A cluster of tuberculosis among crack house contacts in San Mateo County, California. Am J Public Health 1994; 84:1834-6. doi: 10.2105/ajph.84.11.1834.

23. Reyes JC, Robles RR, Colón HM, Marrero CA, Castillo $\mathbf{X}$, Meléndez M. Mycobacterium tuberculosis infection among crack and injection drug users in San Juan, Puerto Rico. P R Health Sci J 1996; 15:233-6.

24. Story A, Bothamley G, Hayward A. Crack cocaine and infectious tuberculosis. Emerg Infect Dis 2008; 14:1466-9. doi: 10.3201/eid1409.070654.

25. do Valle Leone de Oliveira SM, Ferreira da Silva E, Coimbra Motta-Castro AR, de Oliveira Landgraf de Castro V, Stábile AC, Mello Miranda Paniago A, et al. Tuberculosis infection among cocaine crack users in Brazil. Int J Drug Policy 2018; 59:24-7. doi: 10.1016/j.drugpo.2018.06.012.

26. Baldwin GC, Tashkin DP, Buckley DM, Park AN, Dubinett SM, Roth MD. Marijuana and cocaine impair alveolar macrophage function and cytokine production. Am J Respir Crit Care Med 1997; 156:1606-13. doi: 10.1164/ajrccm. 156.5.9704146.

27. Roth MD, Whittaker K, Salehi K, Tashkin DP, Baldwin GC. Mechanisms for impaired effector function in alveolar macrophages from marijuana and cocaine smokers. J Neuroimmunol 2004; 147:82-6. doi: 10.1016/j.jneuroim. 2003.10.017.

28. Observatorio Uruguayo de Drogas. Pasta Base de Cocaína en Uruguay. Compilación. Montevideo: OUD, 2014. Disponible en: https://www.gub.uy/junta-nacional-drogas/sites/junta-nacional-drogas/files/2018-01/Pasta_Base_en Uruguay_Compilacion_0.pdf [Consulta: 4 agosto 2020].

29. Silva MR, Pereira JC, Costa RR, Dias JA, Guimarães MDC, Leite ICG. Drug addiction and alcoholism as predictors for tuberculosis treatment default in Brazil: a prospective cohort study. Epidemiol Infect 2017; 145:3516-24. doi: $10.1017 /$ S0950268817002631

30. Madeira de Oliveira S, Altmayer S, Zanon M, Alves Sidney-Filho L, Schneider Moreira AL, de Tarso Dalcin P, et al. Predictors of noncompliance to pulmonary tuberculosis treatment: an insight from South America. Plos One 2018; 13:e0202593. doi: 10.1371/journal.pone.0202593.

\section{Contribución de autores}

Matilde Outeda, https://orcid.org/0000-0002-1332-4579. Concepción, ejecución, interpretación, revisión de bibliografía y redacción. Elizabeth Marchissio, https://orcid.org/0000-0002-5301-3601. Ejecución, interpretación, revisión de bibliografía. Ivana Labadie, https://orcid.org/0000-0001-6300-017X. Ejecución, interpretación, revisión de bibliografía.

Alice Bica, https://orcid.org/0000-0003-1622-0297. Ejecución, interpretación.

Pilar Gadea, https://orcid.org/0000-0003-0347-8061. Interpretación, redacción y revisión crítica.

Verónica Seija, https://orcid.org/0000-0002-3585-9259. Concepción, diseño, interpretación, redacción y revisión crítica. 